\title{
Medication Reconciliation and Patient Education at a Primary Care \\ Background \\ Office in South Florida \\ Methods
}

Drug prescription errors constitute a significant problem that results in adverse health events. Moreover, discrepancies in drug prescription lists caused during transition from paper to electronic health records increase the likelihood of dangerous health outcomes. Besides, low treatment adherence is an issue that deteriorates this situation. In particular, it is identified that the Hispanic patients are characterized by lower medication adherence (Green et al., 2017). Hence, this group of patients demonstrates better responsiveness to treatment plans in a case when there is a strong nurse-patient connectedness (Sobel et al.,

2016). Consequently, to improve

pharmacovigilance of patients it is necessary to develop patient-provider relations that are characterized by higher level of involvement and health monitoring (Brown \& Bahri, 2019).

\section{Purpose}

The aim of the present DNP project is to enhance pharmacovigilance in Hispanic populations on polypharmacy by conducting a telephone-based monitoring of their drug prescription adherence combined with educational interventions delivered to patients and their family members.
Maria Elena Nasiff, DNP,APRN, ANP-BC, FNP, MSN.,

Telephone-based open-ended structured interviewing aimed at identifying patients'

Dr. Arturo Espinal, MD, Internal Medicine, Adults/Pediatrics., knowledge about therapeutic effects, doses, drugDr. Dana Sherman, DNP, APRN, ANP-BC, FNP-BC.

Nicole Wertheim College of Nursing and Health Sciences, Florida International University

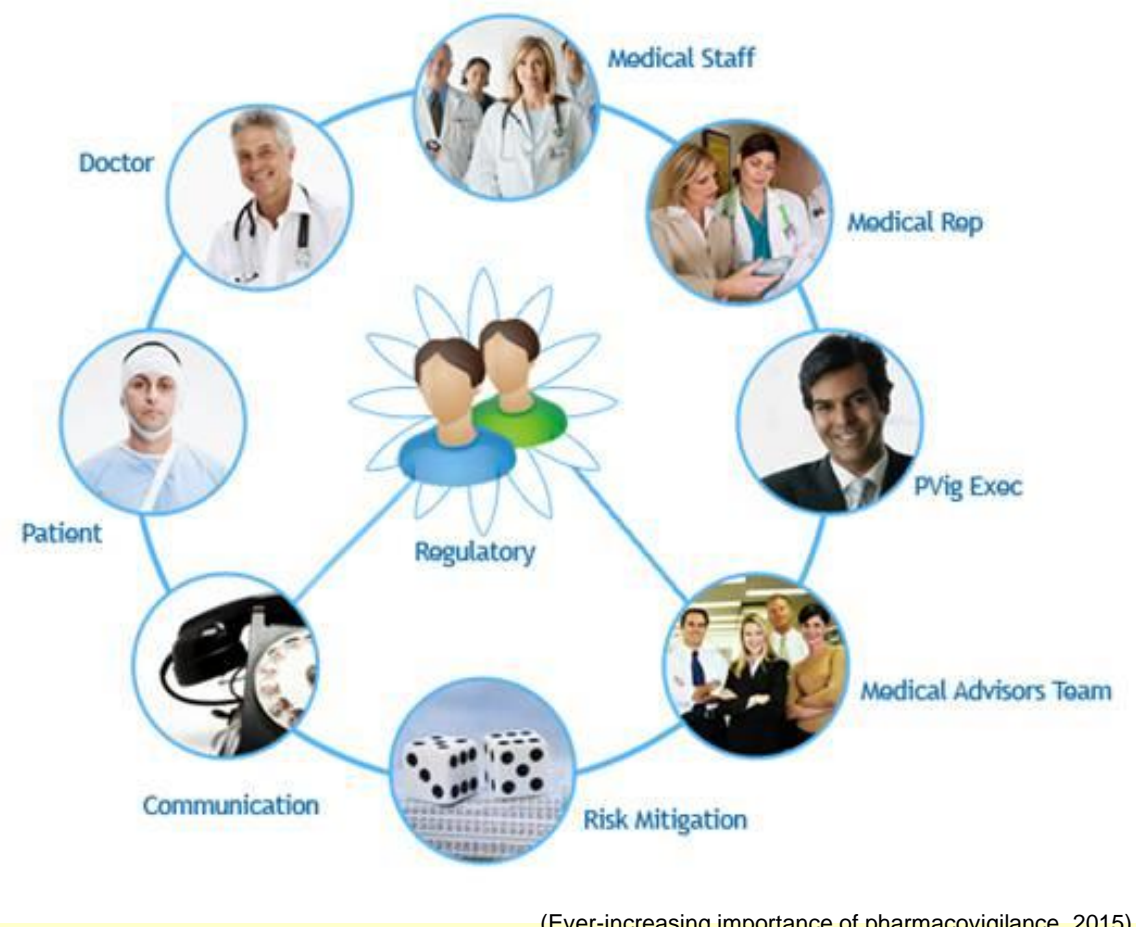
motivational interviews aimed at increasing

healthcare literacy and treatment adherence in the identified group of patients and their families.

\section{Results}

This quality improvement project depicts that the applied method is effective in a short-run perspective.

-Specifically, $100 \%$ of patients answered 'yes' to the control question: 'Do you know why you were prescribed the medications you are taking?'

- $70 \%$ of patients reported the lack of healthcare literacy that was removed during the project while answering the control question: 'What gets in the way of you taking your medication as prescribed?' -Finally, $80 \%$ patients showed advanced selfefficacy and conscious adherence to their treatment plans.

\section{Conclusion}

Conducted quality improvement project sheds light on the effective ways of increasing patients pharmacovigilance by maintaining regular and stronger involved nurse-patient interactions, education, and monitoring of treatment adherence accompanied by family members' support. Hence, this approach is expensive and timeconsuming to be run on a regular basis. Moreover, the sustainability of achieved positive change is unclear in a medium and long-run perspective. 